EPJ manuscript No.

(will be inserted by the editor)

\title{
Backward pion photoproduction
}

\author{
A. Sibirtsev ${ }^{1,2}$, J. Haidenbauer ${ }^{3,4}$, F. Huang ${ }^{3 a}$, S. Krewald ${ }^{3,4}$ and U.-G. Meißner ${ }^{1,3,4}$ \\ 1 Helmholtz-Institut für Strahlen- und Kernphysik and Bethe Center for Theoretical Physics, Universität Bonn, D-53115 Bonn, Germany \\ 2 Excited Baryon Analysis Center (EBAC), Thomas Jefferson National Accelerator Facility, Newport News, Virginia 23606, USA \\ 3 Institut für Kernphysik and Jülich Centre for Hadron Physics, Forschungszentrum Jülich, D-52425 Jülich, Germany \\ ${ }^{4}$ Institute for Advanced Simulations, Forschungszentrum Jülich, D-52425 Jülich, Germany
}

Received: date / Revised version: date

\begin{abstract}
We present a systematic analysis of backward pion photoproduction for the reactions $\gamma p \rightarrow \pi^{0} p$ and $\gamma p \rightarrow \pi^{+} n$. Regge phenomenology is applied at invariant collision energies above $3 \mathrm{GeV}$ in order to fix the reaction amplitude. A comparision with older data on $\pi^{0}$ - and $\pi^{+}$-photoproduction at $\vartheta=180^{\circ}$ indicates that the high-energy limit as given by the Regge calculation could be reached possibly at energies of around $\sqrt{s} \simeq 3 \mathrm{GeV}$. In the energy region of $\sqrt{s} \leq 2.5 \mathrm{GeV}$, covered by the new measurements of $\gamma p \rightarrow \pi^{0} p$ differential cross sections at large angles at ELSA, JLab, and LEPS, we see no clear signal for a convergence towards the Regge results. The baryon trajectories obtained in our analysis are in good agreement with those given by the spectrum of excited baryons.
\end{abstract}

PACS. 11.55.Jy Regge formalism - 13.60.Le Meson production - 25.20.Lj Photoproduction reactions

\section{Introduction}

Recently, backward pion photoproduction in the $\gamma p \rightarrow \pi^{0} p$ reaction attracted significant interest at ELSA [1], JLab [2] and LEPS [3]. New precise data were obtained with the aim to find evidence for high-mass resonances, most of which are not well established [4]. The measurements were done at different angles and up to photon energies that correspond to invariant collision energy of $\sqrt{s} \simeq 2.5 \mathrm{GeV}$ at ELSA and JLab, and $\sqrt{s} \simeq 2.3 \mathrm{GeV}$ at LEPS. As is evident from the publication of the LEPS measurement [3], their data and those collected at ELSA are in disagreement at many of the available energies.

Naturally, the disagreement between the measurements complicates the data evaluation in terms of a partial wave analysis (PWA). For example, the solution of the GWU group [5] does not reproduce the LEPS [3] data at the higher energies. The recent analysis of the GWU group (FA06) presented in [2], which is now extended up to $2.5 \mathrm{GeV}$ and was readjusted to the new CLAS (JLab) data [2], does not describe some of the data from ELSA, noteably at more forward angles [6].

For pion photoproduction at backward angles there is no updated systematic analysis. Actually, the phenomenology of backward photoproduction was last reviewed in 1971 [7]. Thus, in the present paper we want to re-examine the available highenergy data on pion photoproduction at backward angles within the Regge approach. We also consider the very recent measurements from ELSA [1], JLab [2] and LEPS [3]. Thereby we want to clarify to which extent these new data are in line with the result inferred from our Regge fit to the high-energy data.

\footnotetext{
${ }^{\mathrm{a}}$ present address: Dept. of Physics and Astronomy, University of Georgia, Athens, GA 20602, U.S.A.
}

Evidently, with increasing energy the cross sections should approach the high-energy limit [8,9,10], but it is unclear in which energy region this will take place.

We also explore whether the available data exhibit features that could be a signal for high-mass resonance contributions. Indeed, the near-backward direction is the best angular region to find such signals from the excitation of baryons. In this region no appreciable contribution is expected from forward diffractive processes that dominate the reaction in the $t$-channel. For example, the experimental results available for $\pi^{-} p \rightarrow \pi^{-} p$ and $\pi^{+} p \rightarrow \pi^{+} p$ scattering at backward angles [11,12] indicate a sizeable variation of the differential cross section with energy up to $\sqrt{s} \simeq 2.9 \mathrm{GeV}$. This observation might be considered as a direct illustration of the contribution from high-mass resonances to pion-nucleon scattering. Systematic analyses of those reactions at higher energies [11, 12,13,14] showed that from around $3 \mathrm{GeV}$ upwards the data approach the high-energy limit as given by Regge phenomenology. It will be interesting to see whether the situation is similar in case of pion photoproduction at backward angles. Finally, let us note that for reactions at backward angles the Regge approach provides a close connection between the exchange amplitudes and the baryon spectrum.

The paper is structured in the following way: In Sect. 2 we formulate the reaction amplitudes. The baryon trajectories used in our analysis and their properties are discussed in Sect. 3 and Sect. 4, respectively. The results of the fit are presented in Sect. 5. A comparison with data on pion photoproduction at $\vartheta=180^{\circ}$ and other large angles is provided in Sects. 6 and 7. The paper ends with a brief summary. 


\section{Helicity amplitudes}

In the Regge formalism it is convenient to use the $u$-channel parity conserving helicity amplitudes $F_{\lambda}^{ \pm}(\sqrt{u}, s)$ with $\lambda=1,3$ being the net photon-nucleon $u$-channel helicity [9, 15, 16]. Here $s$ is the invariant collision energy squared and $u$ is the squared four-momentum transfered from the photon to the final nucleon. The superscripts indicate the quantum number $P \cdot \mathcal{S}$ of the baryon exchange with $P$ and $\mathcal{S}$ being the parity and signature of the baryon trajectory under consideration, respectively. For the physical particles located on the baryon trajectory the signature factor is defined as $\mathcal{S}=(-1)^{J-1 / 2}$, where $J$ stands for the baryon spin. From that it is clear that the Regge classification of the baryon trajectories that can contribute to the reaction is given in terms of the signature $\mathcal{S}= \pm 1$ and parity $P= \pm 1$ and, therefore, one should consider four trajectories for nucleon- and also for Delta-isobar states. Historically these trajectories are called $\alpha$ (for $\mathcal{S}=1$ and $P=1), \beta(\mathcal{S}=1, P=-1)$, $\gamma(\mathcal{S}=-1, P=-1)$ and $\delta(\mathcal{S}=-1, P=1)$.

The relation between the $u$-channel parity conserving helicity amplitudes $F$ and the standard CGLN invariant amplitudes $\mathcal{F}$ are [17, 18, 19]

$$
\begin{aligned}
F_{1}^{+}(\sqrt{u}, s) & =\frac{i K_{1}}{16 \pi}\left[2\left(u-m_{N}^{2}\right) \mathcal{F}_{1}\right. \\
& +\left(\sqrt{u}+m_{N}\right)\left(t \sqrt{u}-m_{\pi}^{2} m_{N}\right) \mathcal{F}_{2} \\
& +m_{N}\left(t-m_{\pi}^{2}\right)\left(\mathcal{F}_{3}+\mathcal{F}_{4}\right) \\
& \left.+\left(\sqrt{u}+m_{N}\right)\left(u-m_{N}^{2}\right)\left(\mathcal{F}_{3}-\mathcal{F}_{4}\right)\right], \\
F_{3}^{+}(\sqrt{u}, s) & =\frac{i K_{3}}{32 \pi}\left[\left(\sqrt{u}+m_{N}\right) \mathcal{F}_{2}+\mathcal{F}_{3}+\mathcal{F}_{4}\right], \\
F_{1}^{-}(\sqrt{u}, s) & =F_{1}^{+}(-\sqrt{u}, s), \\
F_{3}^{-}(\sqrt{u}, s) & =-F_{3}^{+}(-\sqrt{u}, s),
\end{aligned}
$$

where $K_{1}$ and $K_{3}$ are kinematical factors given by

$$
\begin{aligned}
& K_{1}=\frac{\sqrt{\left(\sqrt{u}-m_{N}\right)^{2}-m_{\pi}^{2}}}{u}, \\
& K_{3}=\frac{\sqrt{\left(\sqrt{u}+m_{N}\right)^{2}-m_{\pi}^{2}}\left[\left(\sqrt{u}-m_{N}\right)^{2}-m_{\pi}^{2}\right]\left(u-m_{N}^{2}\right)}{\sqrt{u} u},
\end{aligned}
$$

with $m_{N}\left(m_{\pi}\right)$ the nucleon (pion) mass. Other relations between the amplitudes defined in various representations and the $u$-channel parity conserving helicity amplitudes can be found in Ref. [20].

The kinematical factors of Eq. (3) contain a singularity at $u=0$. Since the value $u=0$ is within the physical region such a singularity appears in any suitable set of $u$-channel amplitudes used for Reggeization [21,22,23]. The CGLN invariant amplitudes also contain a kinematical singularity at $u=0$ with respect to the Ball amplitudes [19], which satisfy the Mandelstam representation and are analytic functions of $s, t$ and $u$, with $t$ being the squared four-momentum transfer from the initial to the final nucleon.

In order to isolate this kinematical singularity the Regge amplitudes are usually parametrized via certain residue functions that provide $u$-channel amplitudes which vary smoothly over the value $u=0$. The simplest way is to introduce modified amplitudes as in Ref. [23]

$$
\begin{gathered}
\tilde{F}_{1}^{ \pm}(\sqrt{u}, s)=\frac{F_{1}^{ \pm}(\sqrt{u}, s)}{K_{1}( \pm \sqrt{u})}, \\
\tilde{F}_{3}^{ \pm}(\sqrt{u}, s)= \pm \frac{F_{3}^{ \pm}(\sqrt{u}, s)}{K_{3}( \pm \sqrt{u})} .
\end{gathered}
$$

It is clear from Eqs. (2) and (4) that these modified amplitudes satisfy the MacDowell symmetry relations [24]:

$$
\begin{gathered}
\tilde{F}_{1}^{+}(\sqrt{u}, s)=\tilde{F}_{1}^{-}(-\sqrt{u}, s), \\
\tilde{F}_{3}^{+}(\sqrt{u}, s)=\tilde{F}_{3}^{-}(-\sqrt{u}, s) .
\end{gathered}
$$

In practice these symmetry relations allow to reduce the number of parameters used for the Reggeization of the $\tilde{F}$ amplitudes. As will be discussed below the relations in Eq. (5) are significant for the Regge classification of the baryon trajectories. In order to satisfy the MacDowell symmetry, the Regge poles must occur in pairs with opposite parity, with trajectories and residues related by certain conditions listed in the following. These conditions were first discovered by Gribov [25]. Additional constraints for the $u$-channel parity conserving helicity amplitudes are given by a threshold relation [26] that connects the helicity-1/2 and helicity-3/2 amplitudes at $u=m_{N}^{2}$,

$$
\tilde{F}_{1}^{ \pm}\left(m_{N}, s\right)=2 m_{N}\left(t-m_{\pi}^{2}\right) \tilde{F}_{3}^{ \pm}\left(m_{N}, s\right) .
$$

Similar to the particle-exchange Feynman diagram, each amplitude $F$ is factorized in terms of a propagator and a vertex function as [16,22, 23]

$$
\begin{aligned}
& \tilde{F}_{1}^{ \pm}=i \gamma_{1}^{ \pm}(\sqrt{u}) G\left(\alpha^{ \pm}(u), \nu\right), \\
& \tilde{F}_{3}^{ \pm}=i \gamma_{3}^{ \pm}(\sqrt{u}) \frac{\alpha^{ \pm}(\sqrt{u})-1 / 2}{\nu} G\left[\alpha^{ \pm}(u), \nu\right],
\end{aligned}
$$

where the $\gamma$ 's are residue functions that satisfy the symmetry relations of Eq. (5):

$$
\gamma_{1}^{+}(\sqrt{u})=\gamma_{1}^{-}(-\sqrt{u}) \text { and } \gamma_{3}^{+}(\sqrt{u})=\gamma_{3}^{-}(-\sqrt{u}) \text {. }
$$

The invariant variable $\nu$ is defined by

$$
\nu=\frac{s-t}{4 m_{N}}=\frac{2 s+u-2 m_{N}^{2}-m_{\pi}^{2}}{4 m_{N}}
$$

and the Regge propagator is given by

$$
G[\alpha, \nu]=\frac{1+\mathcal{S} \exp [-i \pi(\alpha-1 / 2)]}{\Gamma[\alpha+1 / 2] \cos [\pi \alpha]}\left[\frac{\nu}{\nu_{0}}\right]^{\alpha-1 / 2},
$$

with $\nu_{0}=1 \mathrm{GeV}$. The signature factor $\mathcal{S}$ was already defined previously and $\alpha$ is the Regge baryon trajectory that satisfies also the MacDowell symmetry,

$$
\alpha^{+}(\sqrt{u})=\alpha^{-}(-\sqrt{u})
$$

and was taken as a linear function of $u$,

$$
\alpha(\sqrt{u})=\alpha_{0}+0.9 u
$$


with an intercept $\alpha_{0}$ that is a free parameter. However, as will be illustrated in the next Section, the intercept of the Regge trajectories can also be very well constrained by the classification of the spectrum of excited baryons.

The threshold relation Eq. (6) is explicitly imposed by

$$
\gamma_{1}^{ \pm}=-4 m_{N}^{2}\left(\alpha^{ \pm}-1 / 2\right) \gamma_{3}^{ \pm}-\left(u-m_{N}^{2}\right) \gamma_{0}^{ \pm},
$$

where $\gamma_{0}$ and $\gamma_{3}$ are residue functions, whose parametrizations are given below. Here the $\sqrt{u}$-dependence of the $\gamma$ functions was dropped for simplicity. The residue functions $\gamma_{0}$ and $\gamma_{3}$ as well as the intercept of the baryon trajectories were fitted to the data. We use the following isospin decomposition of the scattering amplitudes for the different charge states:

$$
\begin{aligned}
& \frac{1}{3}\left[N_{V}-\sqrt{3} N_{S}+2 \Delta\right] \text { for } \gamma p \rightarrow p \pi^{0}, \\
& \frac{\sqrt{2}}{3}\left[N_{V}+\sqrt{3} N_{S}-\Delta\right] \text { for } \gamma p \rightarrow n \pi^{+}, \\
& \frac{1}{3}\left[N_{V}+\sqrt{3} N_{S}+2 \Delta\right] \text { for } \gamma n \rightarrow n \pi^{0}, \\
& \frac{\sqrt{2}}{3}\left[N_{V}-\sqrt{3} N_{S}-\Delta\right] \text { for } \gamma n \rightarrow p \pi^{-} .
\end{aligned}
$$

Here $S$ and $V$ are the isoscalar and isovector components of the electromagnetic coupling, respectively. The symbols $N$ and $\Delta$ denote $I=1 / 2$ and $I=3 / 2$ exchange amplitudes. The $N_{S} / N_{V}$ ratio was taken as a constant and, finally, was fitted to the data. The parity-conserving $u$-channel amplitudes $F$ were transformed to the standard $s$-channel helicity amplitudes, $H$, using the matrix relations [20]. The observables are given in terms of the amplitudes $H$ in Refs. [27,28].

\section{Baryon trajectories}

So far we have not discussed what baryon trajectories should be included in the analysis of backward pion photoproduction and we have not specified the functional form of the residue functions. Actually, the functional form of $\gamma_{0}(\sqrt{u})$ and $\gamma_{3}(\sqrt{u})$ can be fixed by the corresponding baryon trajectory to some extent, as will be discussed in the following.

Let us first consider the nucleon trajectory with spin $J=1 / 2$ and with $N(938)$ as the lowest lying state and thus with signature $\mathcal{S}=+1$ and parity $P=+1$. Historically this trajectory is called the $N_{\alpha}$-trajectory. Its contribution to the $F^{+}$amplitudes should be parametrized in terms of the $\gamma_{0}^{+}$and $\gamma_{3}^{+}$residues functions and the $\alpha^{+}$trajectory. The masses of the lowest excited nucleon states with the same quantum numbers $P$ and $\mathcal{S}$, but with higher spins, are listed by the PDG [4] as $F_{15}(1680)$, $H_{19}(2200)$ and $K_{1,13}(2700)$, corresponding to $J=\frac{5}{2}, \frac{9}{2}$ and $\frac{13}{2}$, respectively.

Fig. 1 shows these resonance states in the Chew-Frautschi plot [29,30] of the spin $J$ as a function of $\sqrt{u}=m$, so that $m$ concurs with the masses of the nucleon states listed above at the corresponding $J$ values. The solid line is the Regge trajectory $\alpha^{+}(\sqrt{u})$ given by Eq. (12) with $\alpha_{0}=-0.26$. Here the intercept was simply adjusted by hand but not precisely fitted to the baryon spectrum. Note that in this case $P \cdot \mathcal{S}=+1$.

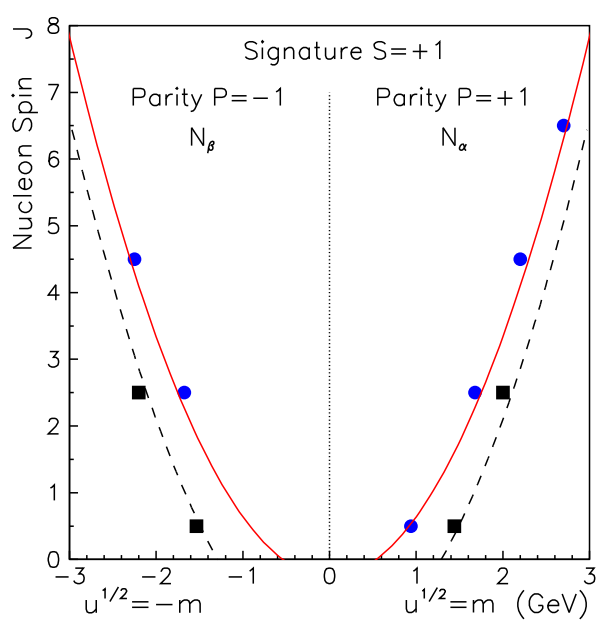

Fig. 1. Chew-Frautschi plot for the $N_{\alpha}$ and $N_{\beta}$ nucleon trajectories indicating the baryon spin $J$ as a function of the mass $(\sqrt{u})$. The results are for the signature $\mathcal{S}=+1$ and for the parities $P= \pm 1$. The circles represent the excited nucleon states listed by the PDG [4] that belong to the leading trajectories. The squares are states that lie on the next-to-leading or so-called daughter trajectories. The solid line indicates the leading trajectories $\alpha^{+}(\sqrt{u})$ and $\alpha^{-}(-\sqrt{u})$ obtained with $\alpha_{0}=-0.26$, while the dashed line corresponds to the daugther trajectories with $\alpha_{0}=-1.5$

The MacDowell symmetry implies the existence of states with the same signature $\mathcal{S}=+1$ but opposite parity $P=-1$. Indeed for such states $P \cdot \mathcal{S}=-1$ and one can apply Eq. (11). The PDG [4] lists the corresponding excited states $D_{15}(1675)$ and $G_{19}(2250)$ for spins $J=\frac{5}{2}$ and $\frac{9}{2}$, respectively. These excited nucleon states are shown in Fig. 1 for $-\sqrt{u}=m$ together with the $\alpha^{-}(-\sqrt{u})$ trajectory. Historically this class of resonances is called $N_{\beta}$-trajectory.

Fig. 11illustrates that, indeed, the symmetry is valid for the existing nucleon resonances with $\mathcal{S}=+1$. But it does not give any explanation why there is no parity partner with $\frac{1}{2}^{-}$, i.e. for the nucleon ground state $\frac{1}{2}^{+}$. Still, this fact must be taken into account in the parameterization of the residue functions [17,25, $31,32]$. Thus, the residues of the trajectory are parametrized as

$$
\begin{array}{r}
\gamma_{0}^{+}(\sqrt{u})=I^{\alpha} \beta_{0}^{\alpha}\left(1+\frac{\sqrt{u}}{0.938}\right), \\
\gamma_{3}^{+}(\sqrt{u})=I^{\alpha} \frac{\beta_{3}^{\alpha}}{m_{N}^{2}-u}\left(1+\frac{\sqrt{u}}{0.938}\right),
\end{array}
$$

where $I^{\alpha}$ is an isospin factor, which defines the contribution to the different reaction channels for photoproduction of the $\pi^{0}$, $\pi^{+}$and $\pi^{-}$-mesons. The constants $\beta_{0}^{\alpha}$ and $\beta_{3}^{\alpha}$ should be fixed by a fit to the data. Note that the residue of Eq. (15) vanishes for the $\frac{1}{2}^{-}$state, i.e. at $\sqrt{u}=-m_{N}$, so that the trajectory does not pass through an unobserved state.

The other nucleon states with $\mathcal{S}=+1$ and $P= \pm 1$ but with higher masses are classified as daughter or granddaughter (etc.) trajectories. The squares in the Fig. 1 show the states $P_{11}(1440)$ and $F_{15}(2000)$ for positive parity and spin $J=\frac{1}{2}$ and $J=\frac{5}{2}$, respectively. The negative parity resonance states in this case are $S_{11}(1535)$ and $D_{15}(2200)$ for $J=\frac{1}{2}$ and $\frac{5}{2}$, respectively. The 


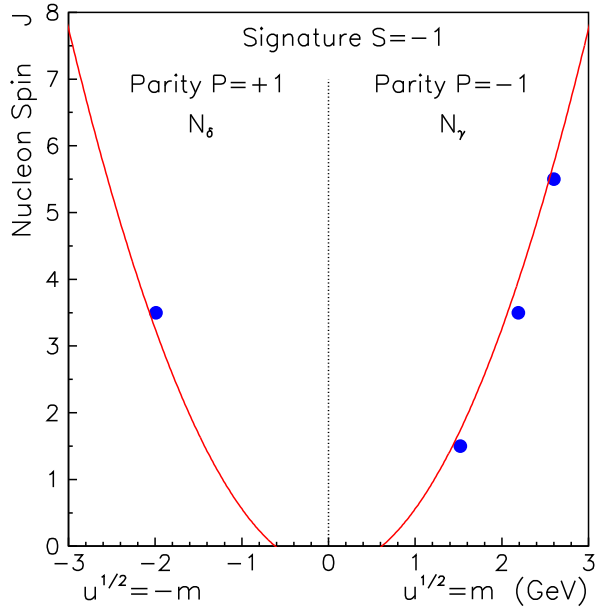

Fig. 2. Chew-Frautschi plot for the $N_{\gamma}$ and $N_{\delta}$ nucleon trajectories indicating the spin baryon $J$ as a function of the the mass $(\sqrt{u})$. The results are for the signature $\mathcal{S}=-1$ and for the parities $P= \pm 1$. The circles represent the excited nucleon states listed by PDG [4]. The line indicates the trajectories $\alpha^{+}(\sqrt{u})$ and $\alpha^{-}(-\sqrt{u})$ for parities $P=-1$ and $P=+1$, respectively, obtained with $\alpha_{0}=-0.34$.

dashed line in Fig. 1 is the linear (next-to-leading) Regge trajectory with $\alpha_{0}=-1.5$.

The secondary (daugther) trajectory is low-lying with respect to the leading trajectory (solid line). Because of the large negative value of $\alpha_{0}$ its contribution to the reaction amplitude is expected to be suppressed due to the $\nu$-dependence of the Regge propagator, cf. Eq. (10). That is why the contributions from secondary trajectories are frequently neglected in Regge analyses. But one should keep in mind that there is no solid argument in favor of such an expectation, because the residue constants $\beta_{0}^{\alpha}$ and $\beta_{3}^{\alpha}$ are free (phenomenological) parameters and could be large.

Now let us consider the nucleon states with signature factor $\mathcal{S}=-1$. For parity $P=-1$ the PDG [4] lists the following resonances: $D_{13}(1520), G_{17}(2190)$ and $I_{1,11}(2600)$ with spin $J=\frac{3}{2}, \frac{7}{2}$ and $\frac{11}{2}$, respectively. These excited nucleons belong to the so-called $N_{\gamma}$-trajectory and are shown in Fig. 2 The line represents the $\alpha^{+}(\sqrt{u})$ trajectory with intercept $\alpha_{0}=-0.34$.

For the $N_{\delta}$-trajectory with $\mathcal{S}=-1$ and parity $P=+1$ the PDG lists $F_{17}(1990)$ with spin $\frac{7}{2}$. There is no indication for a positive-parity partner for the $D_{13}(1520)$ resonance (which has negative parity). This is taken into account in the residue functions for the $N_{\gamma}$-trajectory, which are related to the residues of the $N_{\delta}$-trajectory by the MacDowell symmetry. Namely, we parametrize the residues by

$$
\gamma_{i}^{+}(\sqrt{u})=I^{\gamma} \beta_{i}^{\gamma}\left(1+\frac{\sqrt{u}}{1.52}\right),
$$

for $i=0,3$ with the constants $\beta_{0}^{\gamma}$ and $\beta_{3}^{\gamma}$ as free parameters.

There are only a few known nucleon states that might be discussed in terms of the secondary trajectories with negative signature. Therefore we do not address this question here.

We proceed further with the spectrum of the $\Delta$-resonances and the relevant trajectories. For signature $\mathcal{S}=-1$ and positive parity $P=+1$ the PDG [4] lists the following baryonic

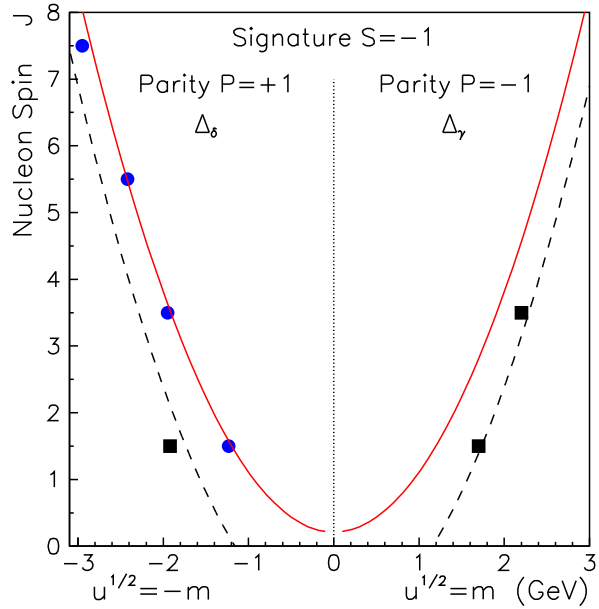

Fig. 3. Chew-Frautschi plot for the $\Delta_{\gamma}$ and $\Delta_{\delta}$ trajectories indicating the spin baryon $J$ as a function of the mass $(\sqrt{u})$. The results are for the signature $\mathcal{S}=-1$ and for the parities $P= \pm 1$. The circles represent excited $\Delta$ states listed by the PDG [4] that belong to the leading trajectory. The solid line indicates the leading trajectories $\alpha^{+}(\sqrt{u})$ and $\alpha^{-}(-\sqrt{u})$ for parities $P=-1$ and $P=+1$, respectively based on the intercept $\alpha_{0}=0.21$. The squares are states that lie on the next-toleading trajectory. The dashed line is the secondary trajectory given by Eq. (12) with $\alpha_{0}=-1.21$.

resonances: $P_{33}(1232), F_{37}(1950), H_{3,11}(2420), K_{3,15}(2950)$ with spin $J=\frac{3}{2}, \frac{7}{2}, \frac{11}{2}$ and $\frac{15}{2}$, respectively. These resonance states form the so-called $\Delta_{\delta}$-trajectory and are shown in Fig. 3 by circles. The solid line is the trajectory $\alpha^{-}(-\sqrt{u})$ with $\alpha_{0}=0.21$. Unfortunately, there are no experimentally well identified $\Delta$ resonances with $\mathcal{S}=-1$ and $P=-1$, which would belong to the $\Delta_{\gamma}$-trajectory and would be the parity partners of the $\Delta_{\delta}$ excited baryons.

So far the partial wave analyses have not found any indication for the $\Delta_{\gamma}$ with a mass around $1.232 \mathrm{GeV}$. Therefore, we parametrize the residue function of the $\Delta_{\delta}$-trajectory by

$$
\gamma_{i}^{-}(-\sqrt{u})=I^{\delta} \beta_{i}^{\delta}\left(1-\frac{\sqrt{u}}{1.232}\right),
$$

for $i=0,3$ and with the constants $\beta_{0}^{\delta}$ and $\beta_{3}^{\delta}$ as free parameters. Note that this parameterization eliminates the lowest-lying $\Delta_{\gamma}$ state, although it is not clear whether the other high-mass baryons of this trajectory indeed exist in nature.

The squares in Fig. 3 show the states lying on next-toleading trajectory. The dashed lines is the secondary trajectory given by Eq. (12) with intercept $\alpha_{0}=-1.21$.

Finally we complete this short review of the nucleon trajectories with the $\Delta$-resonances that have positive signature $\mathcal{S}=+1$. For $P=-1$ we consider the $S_{31}(1620)$ and $D_{35}(1930)$ states with spin $J=\frac{1}{2}$ and $\frac{5}{2}$, respectively. The Regge trajectory containing these resonances is called $\Delta_{\beta}$-trajectory. For the parity $P=+1$ the PDG [4] lists one relevant resonance, the $F_{35}(2000)$, which belongs to the $\Delta_{\alpha}$-trajectory. These excited baryons are shown in the Fig. 4 by circles. The lines are the Regge trajectories with $\alpha_{0}=-1.4$.

Because of the large negative intercept of the $\Delta_{\alpha}$ and $\Delta_{\beta}$ trajectories, their contribution to the total reaction amplitude is 


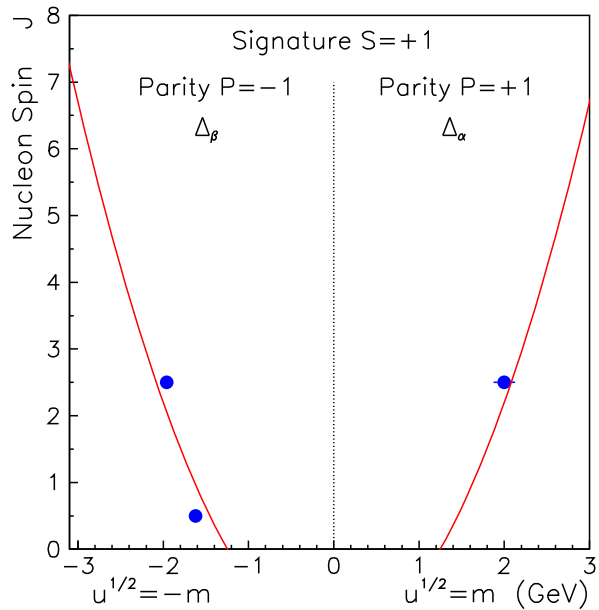

Fig. 4. Chew-Frautschi plot for the $\Delta_{\alpha}$ and $\Delta_{\beta}$ trajectories indicating the baryon spin $J$ as a function of the mass $(\sqrt{u})$. The results are for the signature $\mathcal{S}=+1$ and for the parities $P= \pm 1$. The circles represent the excited states listed by the PDG [4] that belong to the leading trajectory. The solid lines are the trajectories $\alpha^{+}(\sqrt{u})$ and $\alpha^{-}(-\sqrt{u})$ for parities $P=-1$ and $P=+1$, respectively, with intercept $\alpha_{0}=-1.4$.

substantially suppressed due to the $\nu$-dependence of the Regge propagator, cf. Eq. (10). That is the reason why in many analyses these trajectories were not considered.

\section{Propagator properties}

The baryon trajectories roughly determined from the nucleon and $\Delta$-resonance spectra allow for some further constraints on their respective contributions to the reaction amplitude. Let us consider the squared Regge propagator in Eq. (10) and take into account that

$$
\Gamma\left[z+\frac{1}{2}\right] \cos [\pi z]=\pi \Gamma^{-1}\left[\frac{1}{2}-z\right] .
$$

For the trajectories with signature factor $\mathcal{S}= \pm 1$ the squared propagator is

$$
|G|^{2}=\frac{\Gamma^{2}}{\pi^{2}}\left[\frac{1}{2}-\alpha(\sqrt{u})\right]\left[2+2 \mathcal{S} \cos \left[\pi\left(\alpha(\sqrt{u})-\frac{1}{2}\right)\right]\right]\left[\frac{\nu}{\nu_{0}}\right]^{2 \alpha-1}
$$

An interesting feature of the Regge propagator are the zeros of Eq. (19). The zeros at $u \geq 0.6 \mathrm{GeV}^{2}$ have no physical meaning because they are located outside of the scattering (physical) region. This is illustrated in Fig. [5 which shows the correspondence between the scattering angle $\vartheta$ and the four-momentum transfer squared $u$ for different invariant collision energies $\sqrt{s}$ or photon energies $E_{\gamma}$. Here we define the scattering angle in the $s$-channel, i.e. as the pion production angle with respect to the photon beam direction given in the center-of-mass $(\mathrm{cm})$ system. The maximal value of $u$ corresponds to pion photoproduction at the angle $\vartheta=180^{\circ}$.

Fig. 5 makes clear that the squared four-momentum $u$ can be positive as well as negative and thus one indeed has to care

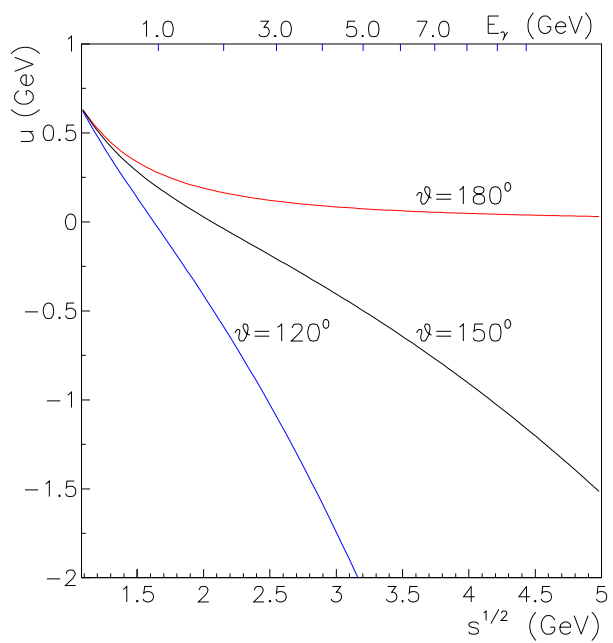

Fig. 5. Four-momentum transfer squared $u$ as a function of the invariant collision energy $\sqrt{s}$ (lower axis) or photon energy (upper axis) for different pion production angles $\vartheta$ (in the $s$-channel).

Table 1. Leading Regge baryon trajectories with signature factor $\mathcal{S}$ and parity $P$. The listed intercepts $\alpha_{0}$ are determined from the spectrum of the excited baryons. The zeros of the squared Regge propagator as given in Eq. $\left[19\right.$, i.e. the values where $|G(u)|^{2}=0$, are listed, too.

\begin{tabular}{|r|r|r|r|r|r|r|}
\hline & $\mathcal{S}$ & $P$ & $\alpha_{0}$ & \multicolumn{3}{|c|}{$\begin{array}{l}\text { Zeros } \\
\mathrm{u}\left(\mathrm{GeV}^{2}\right)\end{array}$} \\
\hline$N_{\alpha}$ & +1 & +1 & -0.26 & -2.49 & -0.26 & 1.96 \\
$N_{\gamma}$ & -1 & -1 & -0.34 & -3.51 & -1.85 & 0.93 \\
$\Delta_{\delta}$ & -1 & +1 & 0.21 & -4.12 & -1.9 & 0.32 \\
$\Delta_{\beta}$ & +1 & -1 & -1.4 & -3.4 & -1.2 & 1.0 \\
\hline
\end{tabular}

about the kinematical singularities at the $u=0$ line. The zeros of the propagator of Eq. (10) at negative four-momentum transfer squared are accessible and might be reflected in scattering observables.

The zeros and the intercepts of the various trajectories are summarized in Table 1 The $N_{\alpha}$-trajectory has its first zero at $u=-0.26 \mathrm{GeV}^{2}$. Indeed, the differential cross sections of the $\pi N \rightarrow \pi N$ reaction exhibit a dip in that $u$ region, though around $u \simeq-0.15 \mathrm{GeV}^{2}[13,33,34,35]$, which does not agree that well with the value of the trajectory.

The pion photoproduction data on differential cross sections show no dip at $|u|<1 \mathrm{GeV}^{2}$. This means that other trajectories than $N_{\alpha}$ and $\Delta_{\beta}$ must dominate the reaction. The $\Delta_{\delta}$ trajectory alone cannot dominate the reaction either because then the cross-section ratio of $\gamma p \rightarrow \pi^{0} p$ to $\gamma p \rightarrow \pi^{+} n$ should be equal to two at small $|u|$. The experimental data indicate that this ratio is close to one at high energies. This also excludes that one can achieve a description of backward pion photoproduction by considering only the $N_{\alpha}$ and $\Delta_{\delta}$ trajectories. Consequently, one has to take into acount also the contribution from the $N_{\gamma}$-trajectory in the Regge analysis of backward scattering.

It is interesting to note that both $N_{\gamma}$ and $\Delta_{\delta}$ trajectories have their first zeros for negative $u$-values around $u \simeq-1.9 \mathrm{GeV}^{2}$. 


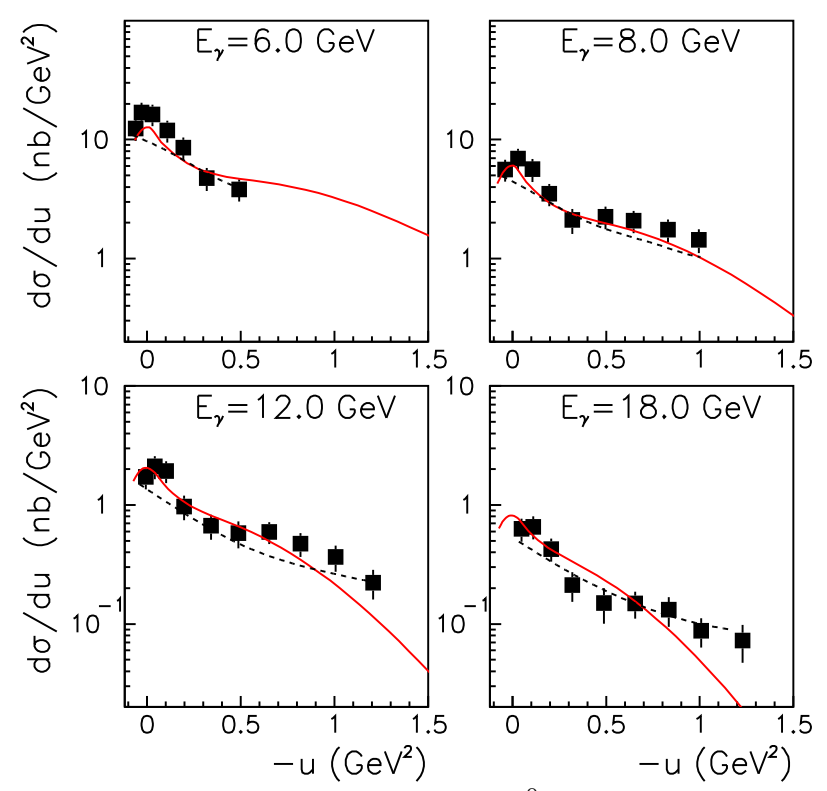

Fig. 6. Differential cross section for $\gamma p \rightarrow \pi^{0} p$ as a function of the fourmomentum transfer squared $u$ at different photon energies $E_{\gamma}$. The squares are SLAC data from Ref. [36]. The solid lines are the results of our Regge model employing the parameters listed in Table 2 The dashed lines are the calculations from Ref. [20].

However, the available data are not precise enough to see whether this feature of the Regge propagator is actually reflected in the observables.

\section{Results at high energies}

Unfortunately, there are not that many data points available at energies above $\sqrt{s} \simeq 3 \mathrm{GeV}$ and for backward angles. Some experimental information on the $\gamma p \rightarrow \pi^{0} p$ differential cross section obtained at SLAC as a function of $u$ is provided in Ref. [36]. Those data points are shown in Fig. 6. The SLAC measurements on the $\gamma p \rightarrow \pi^{+} n$ differential cross section as a function of the squared four-momentum $u$ were published in Refs. [37, [38]. They are presented in Fig. 7 All data points in the range $-u<1 \mathrm{GeV}^{2}$ were included in the fit.

The solid lines in Figs. 6 and 7 represent the results of the fit obtained with the parameters listed in Table 2 Although, in principle, one could use the intercepts of the baryon trajectories extracted from the baryon spectra, we treated them as free parameters. Furthermore, since it turned out that the data are insensitive to the $\Delta_{\beta}$-contribution we did not include this trajectory. The overall $\chi^{2}$ achieved amounts to $\chi^{2} / \mathrm{ndf}=1.2$. It

Table 2. Parameters of the model. The ratio of $N_{S} / N_{V}$ is $-0.08 \pm 0.02$.

\begin{tabular}{|c|c|c|c|}
\hline & $\gamma_{0}$ & $\gamma_{3}$ & $\alpha_{0}$ \\
\hline$N_{\alpha}$ & $72.2 \pm 5.9$ & $-11.6 \pm 3.1$ & $-0.30 \pm 0.09$ \\
$N_{\gamma}$ & $0.54 \pm 2.9$ & $-11.6 \pm 0.4$ & $-0.40 \pm 0.07$ \\
$\Delta_{\delta}$ & $-4.7 \pm 0.9$ & $4.0 \pm 0.2$ & $0.25 \pm 0.02$ \\
\hline
\end{tabular}
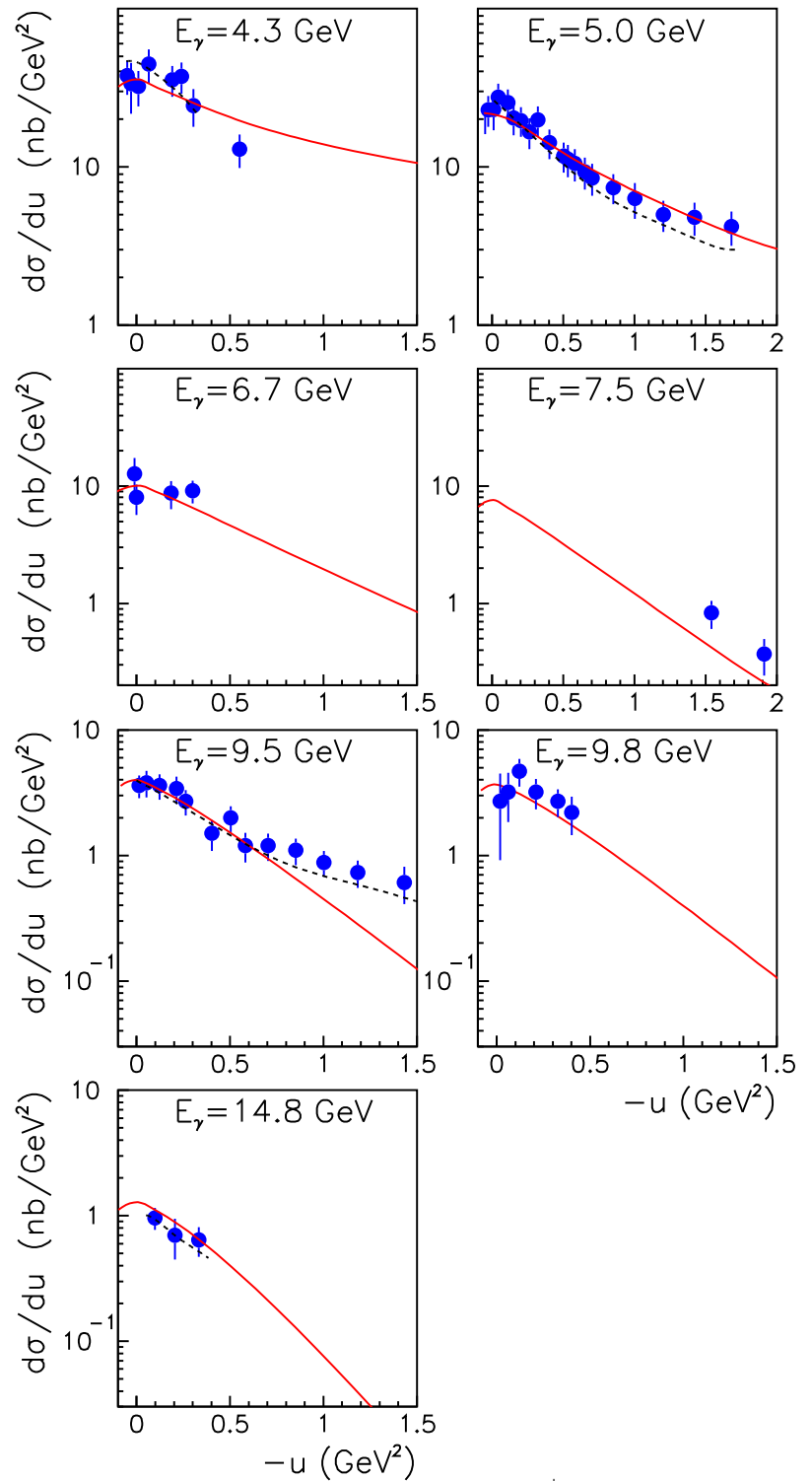

Fig. 7. Differential cross section for $\gamma p \rightarrow \pi^{+} n$ as a function of the four-momentum transfer squared $u$ at different photon energies $E_{\gamma}$. The circles are data from Refs. [37 38]. The solid lines are the results of our Regge model. The results of Ref. [20] (dashed lines) are included at the energies where they are available.

is interesting that the $N_{\alpha}, N_{\gamma}$ and $\Delta_{\delta}$ trajectories determined in the fit to the scattering data imply intercept parameters that are compatible with those extracted from the baryon spectra, cf. Tables 1 and 2. But the partly significant uncertainties of the intercepts obtained in the fit, listed in Table 2 too, indicate that their values are not so well constrained by the backward-angle scattering data.

The overall description of the data by our Regge model is of comparable quality to those reported in earlier studies [13,20, 22,23]. For the ease of comparison, we include here the results of the (latest) previous analysis of backward pion photoproduc- 


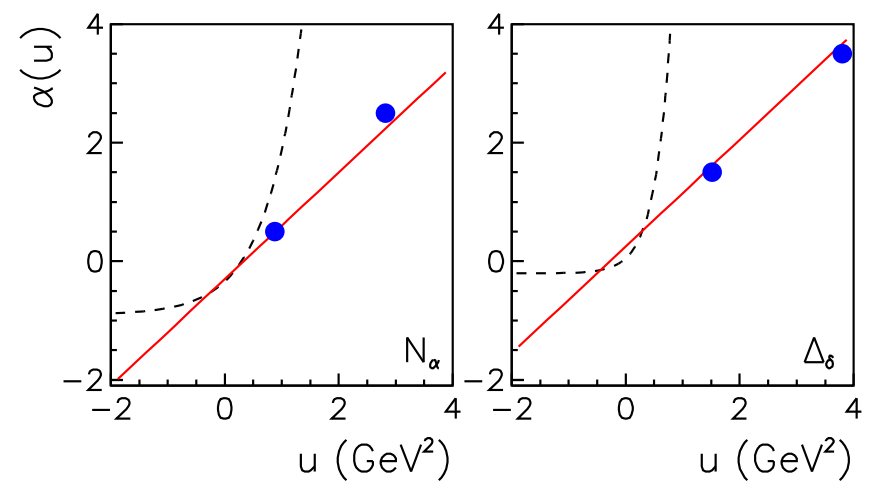

Fig. 8. The trajectories for $N_{\alpha}$ and $\Delta_{\delta}$ exchanges shown as a function of $u$. The solid lines are our parameterizations given by Eq. [12]. The dashed lines indicate the trajectories used in Ref. [20]. The circles show the baryon states with relevant quantum numbers.

tion by Storrow and Triantafillopoulos [20], cf. the dashed lines in Figs. 6 and

The most striking difference in the results is that the model of [20] reproduces the data for larger $-u$ values, i.e. also for $-u>1 \mathrm{GeV}^{2}$, at higher energies. This is due to a principal difference between our approach and the one of Ref. [20] in the parameterization of the exchange trajectories $\alpha(u)$ for the baryon exchanges. While we use linear Regge trajectories (given by Eq. (12) and shown in Fig. 8 by the solid lines for the $N_{\alpha}$ and $\Delta_{\delta}$ exchanges) the trajectories adopted in Ref. [20] are nonlinear functions of the four-momentum transfer squared $u$, as is illustrated by the dashed lines in Fig. 8 Indeed, these nonlinear trajectories, which are modelled by the behaviour of the data, allow them to describe the experiments for $-u>1 \mathrm{GeV}^{2}$, but they are no longer linked to the baryon spectra at $u>0$, cf. Fig. 8. Interestingly, in the region of small $|u|$, where the Regge approach is expected to work best, our results are better in line with the features shown by the data, specifically for the reaction $\gamma p \rightarrow \pi^{0} p$, cf. Fig. 6. For completeness, let us mention that the authors of Ref. [20] also assumed that the $N_{\alpha}$ and $N_{\gamma}$ trajectories are the same.

In other previous works [13] several extensions of the Regge pole model were considered, for example, including absorptive corrections. This again allowed the authors to achieve a description of the data for larger $-u$ values, however, at the expense of practically doubling the number of free parameters that need to be determined in the fit. In view of the scarse data for $-u>1 \mathrm{GeV}^{2}$ we refrain from following that strategy. Note that also some of the model ansatzes considered in [13] have difficulties in reproducing the data at small $|u|$.

Predictions for the polarized photon asymmetry $\Sigma$ are shown in Fig. 9 for two energies.

\footnotetext{
1 We noticed some discrepancies between the data as given in the Durham data base [39], which we use, and the data points drawn in the figures of Ref. [20], especially at photon energy of $5 \mathrm{GeV}$.
}

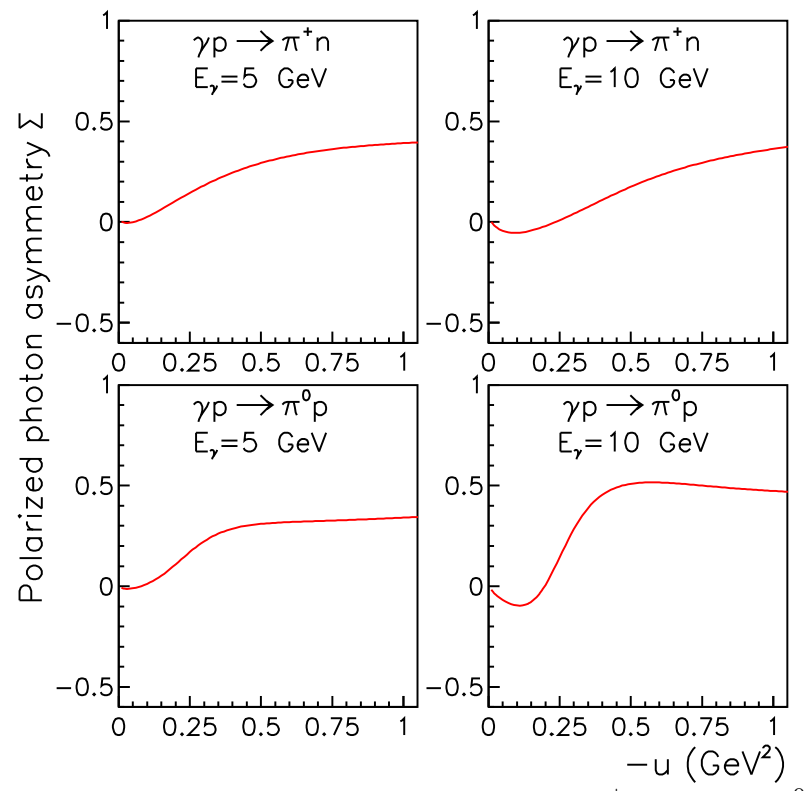

Fig. 9. Polarized photon asymmetry $\Sigma$ for $\gamma p \rightarrow \pi^{+} n$ and $\gamma p \rightarrow \pi^{0} p$ as a function of $-u$, for different photon energies $E_{\gamma}$. The lines are the results of our Regge model.

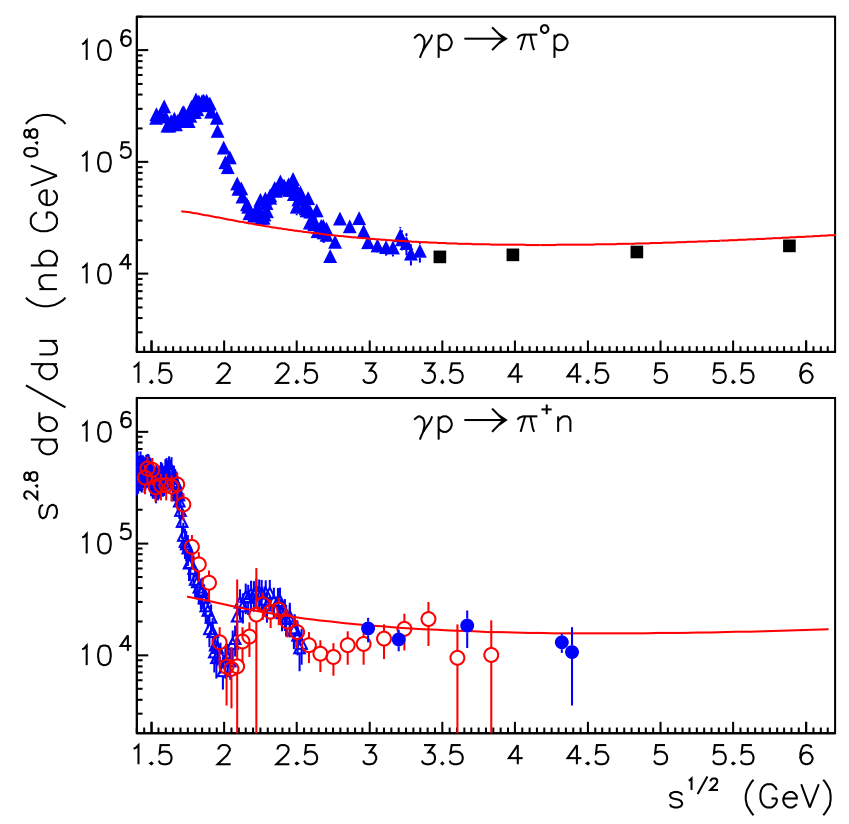

Fig. 10. Differential cross sections for $\pi^{0}$ (upper panel) and $\pi^{+}$(lower panel) photoproduction at $\vartheta=180^{\circ}$ as a function of the invariant collision energy $\sqrt{s}$. Data for the $\pi^{0} p$ channel are from Refs. [40] (triangles) and [36] (squares). Data for the $\pi^{+} n$ channel are from Refs. [41] (open circles), [42] (triangles) and [3738] (filled circles). The lines are the results of our Regge model. Note that all results are multiplied with a factor $s^{2.8}$, cf. text. 


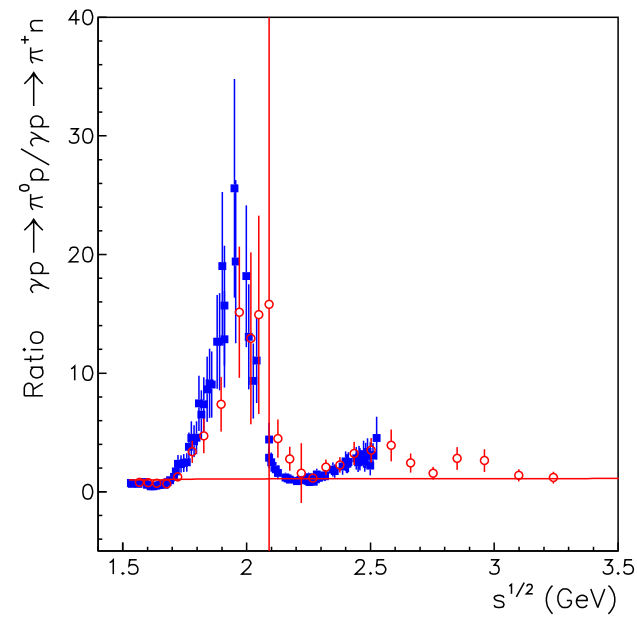

Fig. 11. Ratio of the $\pi^{0}$ to $\pi^{+}$photoproduction cross sections at $\vartheta=180^{\circ}$ as a function of the invariant collision energy. The filled squares are the results obtained with the data from Refs. [40 42] while open circles show the ratios obtained with the data from Refs. [40 41]. The line is the ratio from our Regge model.

\section{Pion photoproduction at $180^{\circ}$}

The experimental information on $\pi^{0}$ - and $\pi^{+}$-meson photoproduction at $\vartheta=180^{\circ}$ is shown in Fig. 10 for a large range of photon energies. Note that the data from Refs. [40] (filled triangles), [41] (open circles) and [42] (open triangles) were not included in our fit, since only few points are available at high energies. The lines in Fig. 10 are the results of our model calculation. We have multiplied the data and also the predictions of our model with a factor $s^{2.8}$ in order to facilitate the comparison between data and theory. This factor corresponds to the energy dependence of the leading Regge trajectory. For energies from around $3 \mathrm{GeV}$ upwards the data seem to approach the high-energy limit inferred from the Regge fit.

At invariant energies from 2 to $3 \mathrm{GeV}$ the data exhibit oscillations around the continuation of the Regge result. It is interesting to observe that the differential cross sections for $\gamma p \rightarrow \pi^{0} p$ and $\gamma p \rightarrow \pi^{+} n$ at $\vartheta=180^{\circ}$ show a very different energy dependence. This is best seen in Fig. 111 where we present the ratio of the $\pi^{0}$ to $\pi^{+}$cross sections at $\vartheta=180^{\circ}$. The line is the ratio obtained for the Regge result which is close to 1.12. The ratio of the experimental values varies strongly with energy and indicates the presence of structures around $\sqrt{s} \simeq 2$ and $2.5 \mathrm{GeV}$. There could be also a structure around $\sqrt{s} \simeq 2.9 \mathrm{GeV}$ but here the accuracy of the data is not sufficient for drawing reliable conclusions.

\section{Photoproduction at large angles}

Differential cross sections for $\gamma p \rightarrow \pi^{+} n$ for several angular ranges in the region $\vartheta>135^{0}$ are shown in Fig. 12 as a function of the invariant collision energy. The data are taken from Refs. [42,45]. The lines indicate the results of our Regge model for $\vartheta=145^{\circ}, 155^{\circ}, 167^{\circ}$, and $180^{\circ}$, respectively. For invariant energies below $2 \mathrm{GeV}$ the data lie above the high-energy limit

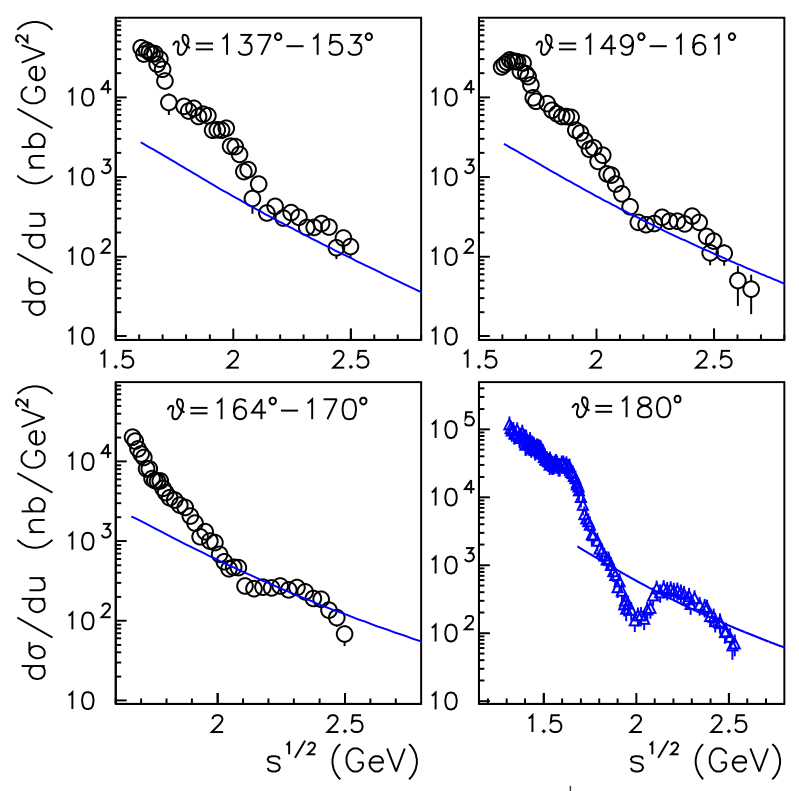

Fig. 12. Differential cross sections for $\gamma p \rightarrow \pi^{+} n$ as a function of the invariant collision energy $\sqrt{s}$, for different production angles $\vartheta$ in the $\mathrm{cm}$ system. The open circles are data from Ref. [45], while the open triangles are from Ref. [42]. The lines are the results of our Regge model.

as given by the Regge calculation. For higher energies the data vary around the Regge predictions and those variations become more pronounced with increasing photoproduction angle. It is interesting to note that at the lowest photoproduction angle considered the data seem to approach the high-energy limit - and even at fairly low energies.

Data on neutral pion photoproduction at large angles were taken at ELSA [1], JLab [2] and LEPS [3]. Fig. 13] contains the data from ELSA. The lines are our Regge results for the $\gamma p \rightarrow \pi^{0} p$ reaction. Here the data lie above the calculations up to somewhat higher energies, i.e. up to $2.2-2.3 \mathrm{GeV}$. It seems that the data approach the high-energy limit in this energy region but reliable conclusions are not possible because of the limited accuracy of the data.

The recent Jefferson Lab data [2] on $\pi^{0}$-meson photoproduction are shown by inverted triangles in Fig. 14t together with the Regge result. Note that in the backward region the JLab measurement extends only up to around $\sqrt{s} \simeq 2.2 \mathrm{GeV}$. At the angles $\vartheta=123^{\circ}$ and $\vartheta=139^{\circ}$ we can compare the data with corresponding experimental information from ELSA [1] (triangles). Obviously, in the kinematical range relevant for our study the two measurements are in reasonable agreement with each other. At last, in Fig. [15 we present the data for $\gamma p \rightarrow \pi^{0} p$ from SPring-8 at LEPS [3] (circles). Also here a comparison with the JLab results [2] is possible at a particular angle $\left(\vartheta=148.2^{0}\right)$, cf. the inverted triangles. These measurements agree nicely with each other, too.

Evidently, the LEPS results do not approach the high-energy limit as inferred from the Regge fit to high-energy data but rather tend to deviate more strongly from the predictions with increasing energy. Thus, at least within the energy region of $\sqrt{s} \leq 2.5 \mathrm{GeV}$, covered by the new measurements of $\gamma p \rightarrow \pi^{0} p$ differential cross sections at large angles [1,2,3], there is no 

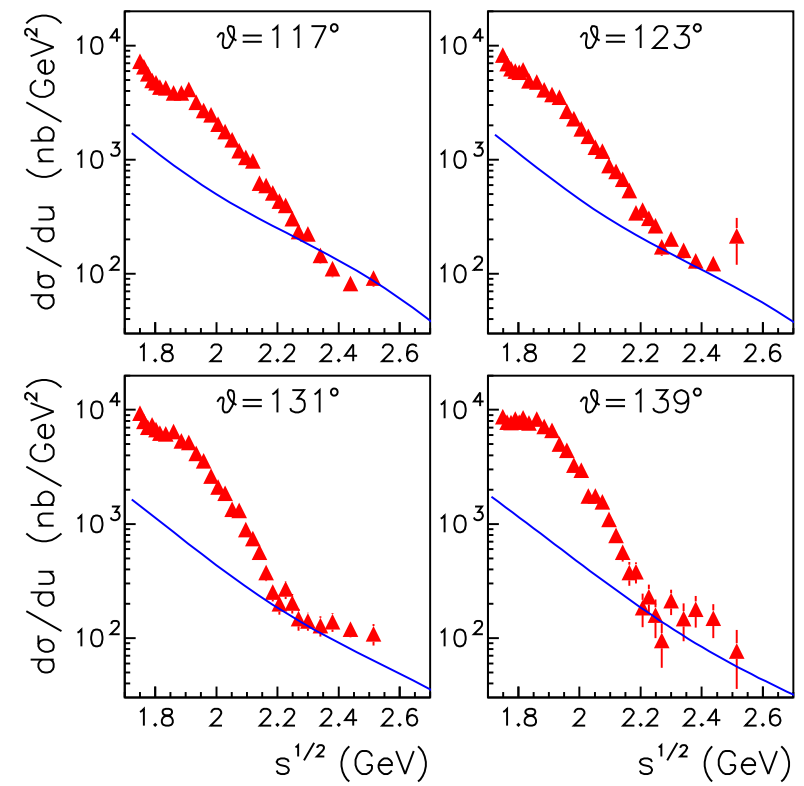

Fig. 13. Differential cross sections for $\gamma p \rightarrow \pi^{0} p$ as a function of invariant collision energy $\sqrt{s}$, for different production angles $\vartheta$ in the $\mathrm{cm}$ system. The data are from the ELSA collaboration [1]. The lines are the results of our Regge model.
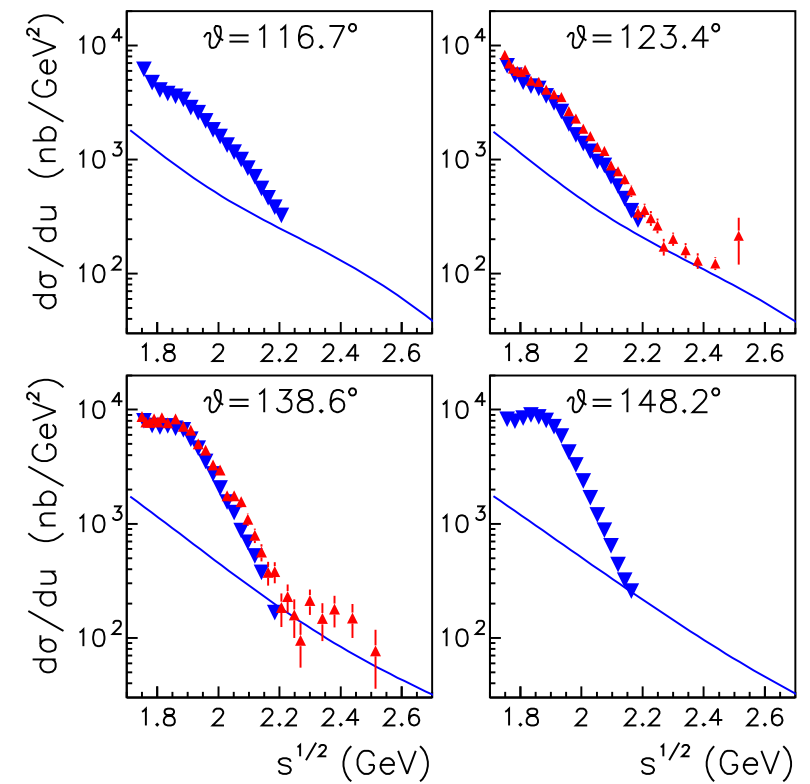

Fig. 14. Differential cross section for $\gamma p \rightarrow \pi^{0} p$ as a function of the invariant collision energy $\sqrt{s}$, for different production angles $\vartheta$ in the $\mathrm{cm}$ system. The inverted triangles are data from JLab [2], while triangles are experimental results from ELSA [1]. The lines are the results of our Regge model.

clear signal for convergence. Therefore, it remains an open question from which energies onwards Regge phenomenology might be applicable. Though the old data [40,41,42] for $\vartheta=180^{\circ}$ that extend to higher energies provide some information with regard to this issue, their precision is not sufficient for drawing reliable conclusions.
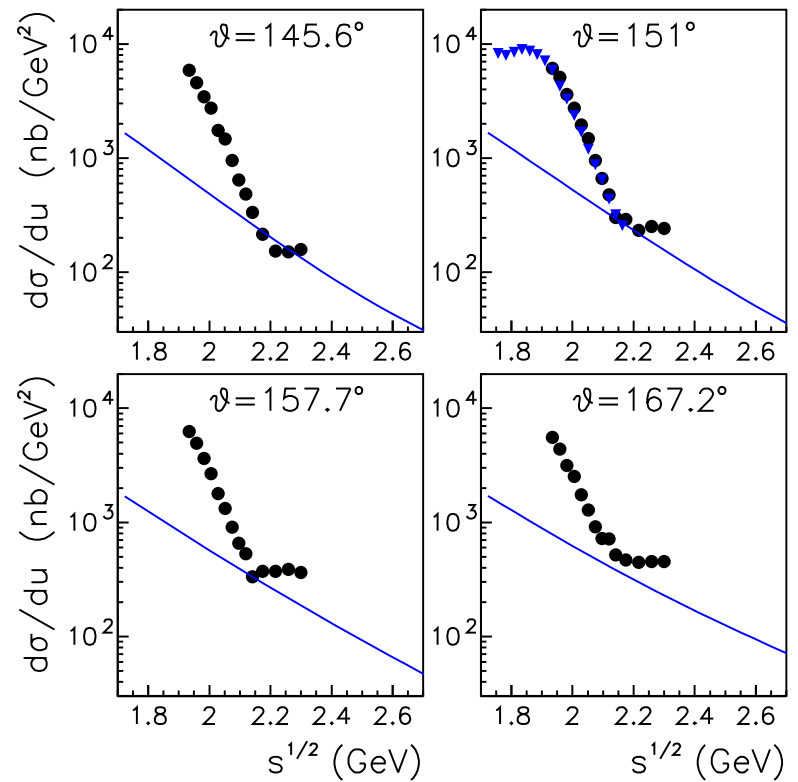

Fig. 15. Differential cross section for $\gamma p \rightarrow \pi^{0} p$ as a function of the invariant collision energy $\sqrt{s}$, for different production angles $\vartheta$ in the $\mathrm{cm}$ system. The solid circles are data from LEPS [3], while the filled inverted triangles are results from JLab [2]. The lines are the results of our Regge model.

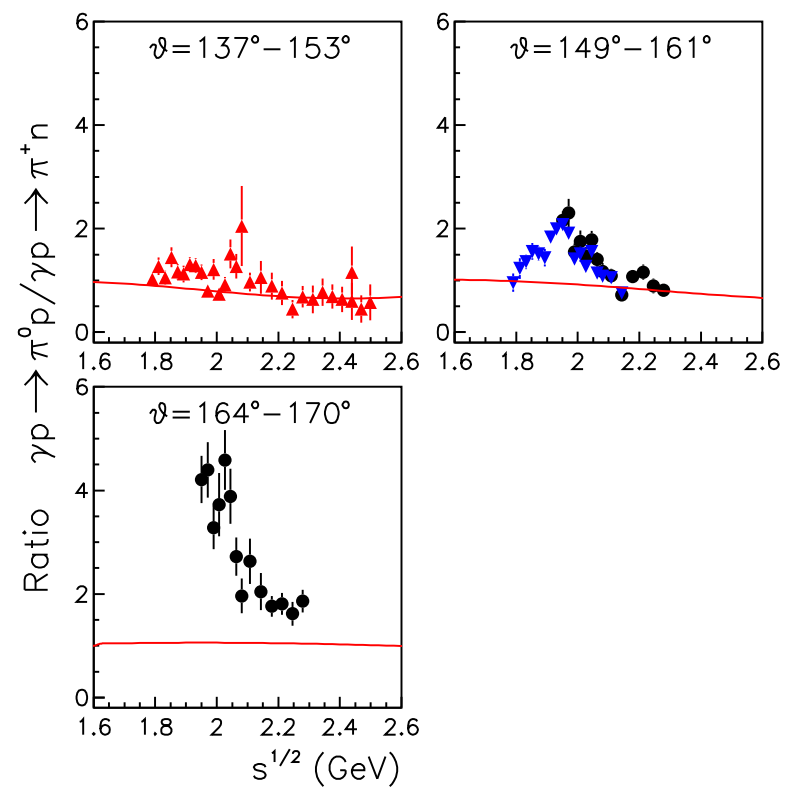

Fig. 16. Ratio of the $\pi^{0}$ to $\pi^{+}$photoproduction cross sections at different angles $\vartheta$ as a function of the invariant collision energy, based on the $\pi^{+}$photoproduction data of [45]. The triangles are the ratio for the $\pi^{0}$ data from ELSA [1], the circles for the LEPS [3] data, and the inverted triangles for the JLab [2] data. The line is the ratio from our Regge model. 


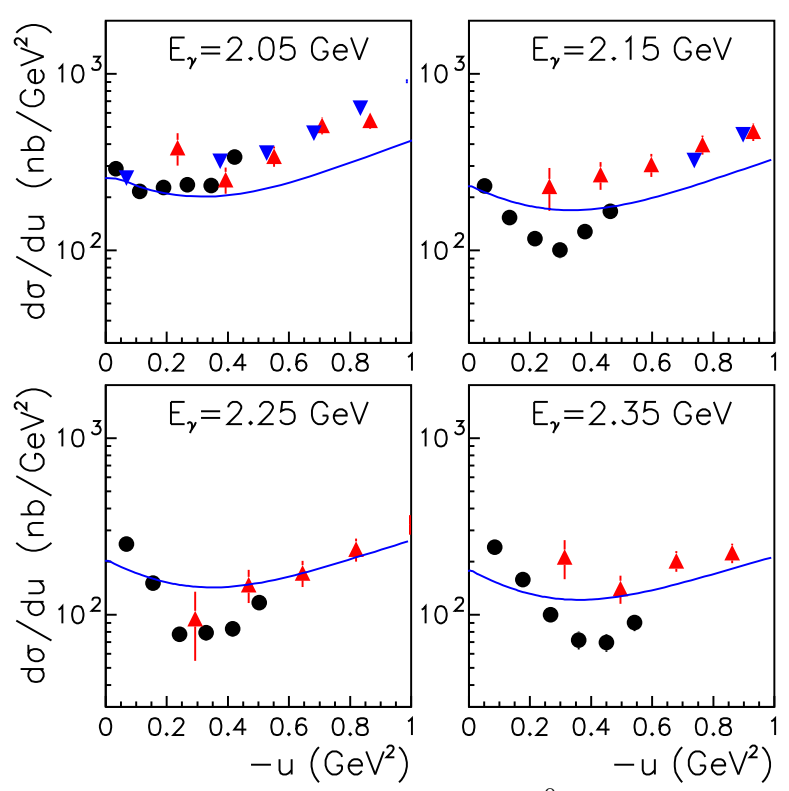

Fig. 17. Differential cross section for $\gamma p \rightarrow \pi^{0} p$ as a function of $-u$, for different photon energies $E_{\gamma}$. The energies indicated in the figures correspond to the invariant collision energies $\sqrt{s} \simeq 2.17,2.22,2.26$ and $2.3 \mathrm{GeV}$, respectively. The triangles are data from ELSA [1], the circles are data from LEPS [3], and the inverted triangles are results from JLab [2]. The lines are the results of our Regge model.

The $\pi^{0}$ and $\pi^{+}$-meson photoproduction cross sections at large angles differ significantly as was already illustrated by the $\pi^{0} / \pi^{+}$cross-section ratio at $\vartheta=180^{\circ}$ in the Fig. 11 Corresponding ratios for other angles in the backward region are presented in Fig. 16. It is interesting to see that at those angles the ratio is much smaller. Specifically, in the range $137^{\circ}-153^{\circ}$ it is even smaller than 2 and, moreover, practically energy independent.

Finally, we compare the $u$ dependence of the data from ELSA, JLab and LEPS at the highest measured energies. The differential cross sections from the different experiments are shown in Fig. 17 in the range $-1 \leq u \leq 0$. The data presented in the figure were taken at almost the same photon energies, corresponding to invariant collision energies in the range from 2.17 to $2.3 \mathrm{GeV}$. Fig. 17 illustrates that the LEPS data are partly in strong disagreement with the ELSA measurement. At the two highest energies the Regge calculation reproduces the ELSA data reasonably well. The LEPS data exhibit a quite different $u$-dependence as compared to the Regge result and they differ significantly in the absolute value too. In any case, one should keep in mind that at such low energies the accessible range is small for $u$ as well as $t$ so that the regions of small $|u|$ and of small $|t|$ tend to overlap. Therefore, one expects that Regge contributions from both channels should come into play and interferences will occur. But in our model only $u$ channel poles are taken into account. Moreover, there are wellestablished (four-star) $N$ - and $\Delta$ resonances in the mass region of 2.19 to $2.42 \mathrm{GeV}$ [4] which should have an impact on the cross sections. Thus, our predictions shown in Fig. 17have primarily an illustrative character.

\section{Summary}

We have performed a systematic analysis of backward photoproduction of pions in the $\gamma p \rightarrow \pi^{0} p$ and $\gamma p \rightarrow \pi^{+} n$ reactions. Regge phenomenology was applied at invariant collision energies above $3 \mathrm{GeV}$ in order to fix the reaction amplitude. The aim of our study was to see whether we can find any clues regarding the energy region where the data approach the highenergy limit as given by the Regge calculation.

The data [40] on $\pi^{0}$-meson photoproduction at $\vartheta=180^{\circ}$ indicate that this limit could be reached possibly at energies of around $\sqrt{s} \simeq 3 \mathrm{GeV}$. The most recent results on neutral pion photoproduction at large angles available from ELSA [1], JLab [2] and LEPS [3] cover energies up to $\sqrt{s}=2.5 \mathrm{GeV}$. Within this energy region there is no clear signal for a convergence towards the results inferred from our Regge fit to high-energy data. Experiments [41,42] on $\pi^{+}$-meson photoproduction at $\vartheta=180^{\circ}$ suggest that in this channel the data might approach the high-energy limit likewise at roughly $\sqrt{s} \simeq 3 \mathrm{GeV}$. However, as in case of $\pi^{0}$ photoproduction, data with higher precision would be needed to allow for a more solid statement.

It is difficult to say whether the new measurements [1, 2,3] indicate any prominent features that could be due to the excitation of high-mass baryons. One expects that backward scattering is the best angular region to find signals from the excitation of baryons. Indeed the experimental results [11,12] available for $\pi p$ scattering at backward angles indicate directly a strong variation of the differential cross section up to invariant energies of $\sqrt{s} \simeq 2.9 \mathrm{GeV}$. Unfortunately, the new backward photoproduction data do not show any such clean features.

We would like to thank T. Nakano and M. Sumihama for sending us the LEPS data. We appreciate discussions with H. Gao, M. Dugger, E. Klempt, E. Pasyuk, U. Thoma. This work is partially supported by the Helmholtz Association through funds provided to the virtual institute "Spin and strong QCD" (VH-VI-231), by the European Community-Research Infrastructure Integrating Activity "Study of Strongly Interacting Matter" (acronym HadronPhysics2, Grant Agreement no. 227431) under the Seventh Framework Programme of EU, and by DFG (SFB/TR 16, "Subnuclear Structure of Matter"). F. H. is grateful for the support from the Alexander von Humboldt Foundation. A.S. acknowledges support by the JLab grant SURA-06-C0452 and the COSY FFE grant No. 41760632 (COSY-085).

\section{References}

1. O. Bartholomy et al., Phys. Rev. Lett.94, 012003 (2005); H. van Pee et al., Eur. Phys. J. A 31, 61 (2007) [arXiv:0704.1776].

2. M. Dugger et al., Phys. Rev. C 76, 025211 (2007) |arXiv:0705.0816].

3. M. Sumihama et al., Phys. Lett. B 657, 32 (2007) [arXiv:0708.1600].

4. C. Amsler et al., Phys. Lett. B 667, 1 (2008).

5. R. Arndt, W.J. Briscoe, I.I. Strakovsky and R.L. Workman, Phys. Rev. C 66, 055213 (2002); http://gwdac.phys.gwu.edu.

6. R. Arndt, W. Briscoe, I. Strakovsky and R. Workman, Eur. Phys. J. A 35, 311 (2008).

7. E.L. Berger and G.C. Fox, Nucl. Phys. B 30, 1 (1971).

8. G. Höhler, Landolt-Börnstein 9, Springer Verlag, Berlin, 1983. 
9. A. C. Irving and R. P. Worden, Phys. Rept. 34, 117 (1977).

10. F. Huang, A. Sibirtsev, S. Krewald, C. Hanhart, J. Haidenbauer and Ulf-G. Meißner, arXiv:0810.2680 Eur. Phys. J. A, in print.

11. V. Barger and D. Cline, Phys. Rev. 155, 1792 (1967).

12. R.R. Crittenden, R.M. Heinz, D.B. Lichtenberg and E. Predazzi, Phys. Rev. D 1, 169 (1970).

13. E.L. Berger and G.C. Fox, Nucl. Phys. B 26, 1 (1970).

14. A. Sibirtsev, J. Haidenbauer, S. Krewald, T.S.H. Lee, Ulf-G. Meißner and A.W. Thomas, Eur. Phys. J. A 34, 49 (2007) arXiv:0706.0183].

15. M. Jacob and G.C. Wick, Ann. Phys. 7, 404 (1959).

16. M. Gell-Mann, M.L. Goldberger, F.E. Low, E. Marx and F. Zachariasan, Phys. Rev. 133, B145 (1964).

17. J.K Storrow, Electromagnetic Interactions of Hadrons 1, edited by A. Donnachie and G. Shaw, Plenum, NY (1978), pp. 263.

18. G.F. Chew, M.L. Goldberger, F.E. Low and Y. Nambu, Phys. Rev. 106, 1345 (1957).

19. J.S. Ball, Phys. Rev. 124, 2014 (1961).

20. J.K Storrow and E. Triantafillopoulos, J. Phys. G 4, 1679 (1978).

21. E.A. Paschos, Phys. Rev. Lett. 21, 1855 (1968).

22. J.V. Beaupre and E.A. Paschos, Phys. Rev. D 1, 2040 (1970).

23. V. Barger and P. Weiler, Nucl. Phys. B 20, 615 (1970).

24. S.W. MacDowell, Phys. Rev. 116, 774 (1959).

25. V.N. Gribov, JETP 16, 1080 (1963).

26. J.D. Jackson and G.E. Hite, Phys. Rev. 169, 1248 (1968).

27. I.S. Barker, A. Donnachie and J.K. Storrow, Nucl. Phys. B 95, 347 (1975).

28. M. Benmerrouche, N.C. Mukhopadhyay and J.F. Zhang, Phys. Rev. D 51, 3237 (1995).

29. V. Barger and D. Cline, Phys. Rev. Lett. 20, 298 (1968).

30. R. Fiore, L.L. Jenkovszky, F. Paccanoni and A. Prokudin, Phys. Rev. D 70, 054003 (2004).

31. P. Minkowski, Lett. Nuovo Cim. 3S1, 503 (1970) [Lett. Nuovo Cim. 3, 503 (1970)].

32. R. Carlitz and M. Kislinger, Phys. Rev. Lett. 24, 186 (1970).

33. V. Barger and D. Cline, Phenomenological Theories of High Energy Scattering, New York, Benjamin (1969).

34. J.K. Storrow and G.A. Winbow, Nucl. Phys. B 54, 560 (1973).

35. J.K. Storrow and G.A. Winbow, J. Phys. G 1, 263 (1975).

36. D.H. Tompkins et al., Phys. Rev. Lett. 23, 725 (1969).

37. R. Anderson et al., Phys. Rev. Lett. 21, 479 (1968).

38. R. Anderson et al., Phys. Rev. D 14, 679 (1976).

39. The Durham HEP Databases, http://durpdg.dur.ac.uk/

40. G. Buschhorn et al., Phys. Rev. Lett. 20, 230 (1968).

41. K. Ekstrand et al., Phys. Rev. D 6, 1 (1972).

42. B. Bouquet et al., Phys. Rev. Lett. 27, 1244 (1971).

43. L.Y. Zhu et al., Phys. Rev. C 71, 044603 (2005) [arXiv:nucl-ex/0409018].

44. L.Y. Zhu et al., Phys. Rev. Lett. 91, 0222003 (2003) [arXiv:nucl-ex/0211009].

45. R.A. Alvarez et al., Phys. Rev. D 1, 1946 (1970).

46. P.J. Bussey et al., Nucl. Phys. B 154, 492 (1979). 